\title{
BETWEENNESS OF PARTIAL ORDERS
}

\author{
Bruno Courcelle*
}

\begin{abstract}
We construct a monadic second-order sentence that characterizes the ternary relations that are the betweenness relations of finite or infinite partial orders. We prove that no first-order sentence can do that. We characterize the partial orders that can be reconstructed from their betweenness relations. We propose a polynomial time algorithm that tests if a finite relation is the betweenness of a partial order.
\end{abstract}

Mathematics Subject Classification. 06A06, 03B10, 03C13, 03C15.

Received April 20, 2020. Accepted November 19, 2020.

\section{INTRODUCTION}

Betweenness is a standard notion in the study of structures such as trees, partial orders and graphs. It is defined as the ternary relation $B(x, y, z)$ expressing that an element $y$ is between $x$ and $z$, in a sense that depends on the considered structure. This relation is easy to understand and axiomatize in first-order (FO) logic for linear orders. In particular, a linear order can be uniquely described, up to reversal, from its betweenness relation. However, the notion of partial betweenness ${ }^{1}$ raises some difficult algorithmic and logical problems ([8], Chap. 9).

Betweenness in partial orders is axiomatized in [10] by an infinite set of FO sentences that cannot be replaced by a finite one, as we will prove. In this article, we axiomatize betweenness in partial orders by a single monadic second-order (MSO) sentence. We characterize the partial orders that are uniquely reconstructible, up to reversal, from their betweenness relations. We show that an MSO formula can describe some partial order $P$ such that $B_{P}=B_{Q}$ from the betweenness relation $B_{Q}$ of a partial order $Q$ : this definition yields a partial order that may be a proper suborder of $Q$. We give a polynomial time algorithm to test if a finite ternary structure is the bewteenness relation of some partial order and to produce relevant partial orders if this is possible.

Several notions of betweenness in graphs have also been investigated and axiomatized. We only refer to the survey [1] that contains a rich bibliography. Another reference is [2]. In previous articles we have studied betweenness in finite or infinite trees, and also in generalized trees, defined as the partial orders such that the set of elements larger than any one is linearly ordered [4-7]. The corresponding betweenness relations are defined from partial orders, but not as in [10] and in the present article.

Keywords and phrases: Betweenness, partial order, axiomatization, monadic second-order logic, comparability graph.

LaBRI, CNRS and Bordeaux University, 33405 Talence, France.

* Corresponding author: courcell@labri.fr

${ }^{1}$ The question is whether a given ternary relation $B$ is included in the betweenness relation of some linear order. This problem is NP-complete. This question should not be confused with the characterization of betweenness of partial orders. 
This work contributes to the understanding of the expressive power of monadic second-order logic in finite and infinite graphs and related relational structures. We refer to $[3,8]$ for monadic second-order logic.

\section{DEFINITIONS AND KNOWN RESUltS}

All partial orders, graphs and relational structures are finite or countably infinite. The cardinality of a set $X$ is denoted by $|X| \in \mathbb{N} \cup\{\omega\}$.

Let $P=(V, \leq)$ be partial order. A chain (resp. an antichain) is a subset $X$ of $V$ that is linearly ordered (resp. where any two elements are incomparable). We say that $P$ is trivial if $V$ is an antichain.

Definition 2.1. Betweenness. To shorten writings $\neq\left(x_{1}, x_{2}, \ldots, x_{n}\right)$ means that $x_{1}, x_{2}, \ldots, x_{n}$ are pairwise distinct.

(a) Betweenness in linear orders

Let $L=(V, \leq)$ be a linear order. Its betweenness relation $B_{L}$ is the ternary relation on $V$ defined by:

$$
B_{L}(x, y, z): \Longleftrightarrow x<y<z \text { or } z<y<x .
$$

The following properties hold for $B=B_{L}$ and all $x, y, z, u \in V$ :

$$
\begin{aligned}
& \text { B1 : } B(x, y, z) \Rightarrow \neq(x, y, z) . \\
& \text { B2 }: B(x, y, z) \Rightarrow B(z, y, x) . \\
& \text { B3 : } B(x, y, z) \Rightarrow \neg B(x, z, y) . \\
& \text { B4: } B(x, y, z) \wedge B(y, z, u) \Rightarrow B(x, y, u) \wedge B(x, z, u) . \\
& \text { B5 : } B(x, y, z) \wedge B(x, u, y) \Rightarrow B(x, u, z) \wedge B(u, y, z) \\
& \text { B6 : } \neq(x, y, z) \Rightarrow B(x, y, z) \vee B(x, z, y) \vee B(y, x, z) .
\end{aligned}
$$

We get an axiomatization by finitely many universal first-order sentences: a ternary structure $S=(V, B)$ satisfies these properties, if and only if $B=B_{L}$ for some linear order $L=(V, \leq)$. We will say that the class of betweenness relations of linear orders is first-order (FO) definable or axiomatizable. If $|V| \geq 3$, the order $L=(V, \leq)$ and its reversal $L^{r e v}:=(V, \geq)$ are the only ${ }^{2}$ partial orders whose betweenness relation is $B_{L}$, see $[4,5,8]$. We will say that $\leq$ is uniquely defined, up to reversal (written u.t.r.), or reconstructible from its betweenness relation.

(b) Betweenness in partial orders

The betweenness relation $B_{P}$ of a partial order $P=(V, \leq)$ - we will also say a poset ${ }^{3}$ - is the ternary relation on $V$ defined, as in (a), by:

$$
B_{P}(x, y, z): \Longleftrightarrow x<y<z \text { or } z<y<x .
$$

We denote by $\operatorname{Bet}(P)$ the ternary structure $\left(V, B_{P}\right)$. For all $x, y, z, u, v \in V$, the relation $B=B_{P}$ satisfies Properties B1 to B5 together with:

$$
\begin{aligned}
& \mathrm{X}: B(x, y, z) \wedge B(u, y, v) \Rightarrow B(x, y, u) \vee B(x, y, v), \\
& \mathrm{F}: B(x, y, z) \wedge B(y, u, v) \Rightarrow B(x, y, u) \vee B(z, y, u),
\end{aligned}
$$

and an infinite set $\mathcal{O}$ of properties expressed by universal first-order sentences. The notation is borrowed to the article by Lihova [10] who proved, conversely, that if a ternary structure $S=(V, B)$ satisfies these properties, then $B=B_{P}$ for a poset $P=(V, \leq)$ and, of course for its reversal $P^{r e v}:=(V, \geq)$. We will prove that no finite set of first-order sentences can characterize betweenness in posets. Our proof will use the following examples.

(c) A B-cycle is a ternary structure $(V, B)$ such that $V=\left\{a_{1}, a_{2}, \ldots, a_{n}, b_{1}, b_{2}, \ldots, b_{n}\right\}, n \geq 2$, and $B$ consists of the triples $\left(a_{1}, b_{1}, a_{2}\right),\left(a_{2}, b_{2}, a_{3}\right), \ldots,\left(a_{n-1}, b_{n-1}, a_{n}\right),\left(a_{n}, b_{n}, a_{1}\right)$ and the inverse ones, $\left(a_{2}, b_{1}, a_{1}\right),\left(a_{3}, b_{2}, a_{2}\right), \ldots$ so that B2 is satisfied. This structure satisfies Properties B1-B5. If $n$ is even, then $B=B_{P}$ where $P=(V, \leq)$ is the poset such that:

\footnotetext{
${ }^{2}$ If $|V|=2$, the trivial order has the same empty betweenness relation.

${ }^{3}$ This is an inelegant but short terminology for partial order, partial ordering or partially ordered set.
} 


$$
a_{1}<b_{1}<a_{2}>b_{2}>a_{3}<b_{3}<a_{4}>\ldots a_{n-1}<b_{n-1}<a_{n}>b_{n}>a_{1},
$$

and no other inequality holds except by transitivity (e.g. $a_{1}<a_{2}$ ). If $n$ is odd, no such partial order does exist (cf. Lem. 4.2). Consider for example the case $n=3$. A partial order $P$ such that $a_{1}<b_{1}$ and $B_{P}=B$ must verify $b_{1}<a_{2}>b_{2}>a_{3}<b_{3}<a_{1}$ but then, we would have $\left(b_{3}, a_{1}, b_{1}\right)$ in $B$, which is not assumed. The set $\mathcal{O}$ excludes these odd $B$-cycles.

(d) If a partial order is trivial or if it has no chain of cardinality more than 2 , then its betweenness relation is empty. It tells nothing about it.

We will prove that the class of betweenness relations of partial orders is monadic second-order (MSO) definable without using the set $\mathcal{O}$. We will also identify the partial orders that can be reconstructed u.t.r. from their betweenness relations, independently of any logical description. We refer to $[3,8]$ for first-order and monadic second-order logic.

Definition 2.2. Comparability graphs and Gaifman graphs. (a) A ternary structure is a pair $S=(V, B)$ such that $B \subseteq V^{3}$. Its Gaifman graph ${ }^{4}$ is $G f(S):=(V, E)$ where there is an undirected edge $u-v$ in $E$ if and only if $u \neq v$ and $u$ and $v$ belong to a same triple in $B$.

We say that $S$ is connected if $G f(S)$ is. If $G f(S)$ is not connected, then $S$ is the union of the pairwise disjoint induced structures $S[X]:=\left(X, B \cap X^{3}\right)$, called the connected components of $S$, where the sets $X$ are the vertex sets of the connected components of $G f(S)$.

(b) If $P=(V, \leq)$ is a partial order, its comparability graph $\operatorname{Comp}(P)$ has vertex set $V$ and an edge $u-v$ if and only if $u$ and $v$ are different and comparable, i.e., $u<v$ or $v<u$. It is the Gaifman graph of the binary structure $P$.

(c) We say that $P$ is connected if $\operatorname{Comp}(P)$ is. If $\operatorname{Comp}(P)$ is not connected, then $P$ is the union of the pairwise disjoint posets $P[X]:=\left(X, \leq \cap X^{2}\right)$ where the sets $X$ are the vertex sets of the connected components of $\operatorname{Comp}(P)$.

We have $G f(\operatorname{Bet}(P)) \subseteq \operatorname{Comp}(P)$. The inclusion may be proper (see Ex. 2.3). If $G f(\operatorname{Bet}(P))$ is connected, then so is $\operatorname{Comp}(P)$, but not necessarily conversely, because $G f(\operatorname{Bet}(P))$ has no edge if $P$ has no chain of cardinality 3 .

Example 2.3. Here is an example where $G f(\operatorname{Bet}(P)) \subset \operatorname{Comp}(P)$. Let $P=(V, \leq)$ where $V=\{a, b, c, d, e, f\}$ and $\leq$ is generated by $a<b<c<d, e<c, e<f, b<f$, reflexivity and transitivity. The edge $e-f$ of $C o m p(P)$ is not in $G f(\operatorname{Bet}(P))$ because $e$ and $f$ do not belong to any chain of cardinality 3. If we remove the clause $e<f$, the resulting partial order $P^{\prime}$ has the same betweenness relation as $P$ and $G f\left(\operatorname{Bet}\left(P^{\prime}\right)\right)=\operatorname{Comp}\left(P^{\prime}\right)$. We will generalize this observation in Proposition 3.3.

\section{BetWEENNESS IN PARTIAL ORDERS}

Definition 3.1. Extremal elements and B-minimality. (a) Let $P=(V, \leq)$ be a partial order. We define $\operatorname{Min}(P)$ and $\operatorname{Max}(P)$ as the sets of, respectively, the minimal and the maximal elements of $P$. They are its extremal elements and $\operatorname{Ext}(P):=\operatorname{Min}(P) \cup \operatorname{Max}(P)$. An element is isolated if it is an isolated vertex of $\operatorname{Comp}(P)$, equivalently, if it belongs to $\operatorname{Min}(P) \cap \operatorname{Max}(P)$.

(b) In a ternary structure $S=(V, B)$ that satisfies Properties B1,B2 and B3 (in order to avoid uninteresting cases), we say that an element $x$ is extremal if $B(y, x, z)$ does not hold for any $y, z$. The extremal elements of a structure $\operatorname{Bet}(P)$ are the extremal elements of $P$. An element is isolated in $S$ if it is in its Gaifman graph. An isolated element of $P$ is isolated in $\operatorname{Bet}(P)$. The converse is true if $P$ is $\mathrm{B}$-minimal.

(c) A poset $P$ is $B$-minimal if $G f(\operatorname{Bet}(P))=\operatorname{Comp}(P)$, equivalently, if every two comparable elements belong to a chain of cardinality at least 3. A trivial poset is B-minimal. So is one without maximal or without minimal elements.

\footnotetext{
${ }^{4}$ This graph is defined similarily for arbitrary relational structures, not only for ternary ones.
} 
(d) A poset $P$ is strongly B-minimal if, for any $x \in \operatorname{Min}(P)$ and $y \in \operatorname{Max}(P)$, we have $x<z<y$ for some $z$. This property implies B-minimality. If $\operatorname{Min}(P)$ or $\operatorname{Max}(P)$ is empty, then $P$ is strongly B-minimal.

Example 3.2. Let $P=(V, \leq)$ where $V=\{a, b, c, d, e, f\}$ and $\leq$ is generated by $a<b<c, d<e<f, d<c$, reflexivity and transitivity. Then $\operatorname{Comp}(P)$ is connected but $G f(\operatorname{Bet}(P))$ is not. It is not B-minimal. The only orderings on $V$ that yield $\operatorname{Comp}(P)$ as comparability graph are $P$ and $P^{r e v}$. The graph $G f(\operatorname{Bet}(P))$ has two connected components with vertex sets $\{a, b, c\}$ and $\{d, e, f\}$. From it, one obtains four orderings on $V$ that yield the betweenness structure $\operatorname{Bet}(P)$. We will develop this observation.

Proposition 3.3. Let $P=(V, \leq)$ be a poset and $\leq^{\prime}$ be the partial order defined from $<^{\prime}$ such that $x<^{\prime} y$ if and only if $x<y$ and, if $x \in \operatorname{Min}(P)$ and $y \in \operatorname{Max}(P)$, then $x<z<y$ for some $z$. Then $\widetilde{P}:=\left(V, \leq^{\prime}\right) \subseteq P$ is a $B$-minimal poset and $\operatorname{Bet}(\widetilde{P})=\operatorname{Bet}(P)$. It is the unique minimal poset $Q=\left(V, \leq_{Q}\right)$ such that $\operatorname{Bet}(Q)=\operatorname{Bet}(P)$ and $Q \subseteq P$.

Proof. If $x<^{\prime} y<^{\prime} z$ then $x<y<z$, hence $x<z$, and if $x \in \operatorname{Min}(P)$ and $z \in \operatorname{Max}(P)$, we have $y$ between them, so that $x<^{\prime} z$. Hence, $\widetilde{P}:=\left(V, \leq^{\prime}\right)$ is actually a poset and $\widetilde{P} \subseteq P$.

We have $\operatorname{Bet}(\widetilde{P}) \subseteq \operatorname{Bet}(P)$. However, if $x<y<z$, we have $x<^{\prime} y<^{\prime} z$ by the definitions. Hence, $\widetilde{P}$ and $P$ have the same chains of cardinality at least 3 . In particular, $\operatorname{Bet}(\widetilde{P})=\operatorname{Bet}(P)$.

If $\widetilde{P}$ is not B-minimal, there are $x, y$ such that $x<^{\prime} y$ and $x$ and $y$ do not belong to any chain of cardinality 3 in $\widetilde{P}$, whence, in $P$. As $x<y$ this implies that $x \in \operatorname{Min}(P)$ and $y \in \operatorname{Max}(P)$, but we have $x<z<y$ for some $z$, hence we have $x<^{\prime} z<^{\prime} y$, which contradicts the assumption that $x$ and $y$ do not belong to a chain of cardinality 3 in $\widetilde{P}$. Hence, $\widetilde{P}$ is B-minimal.

Assume that $Q=\left(V, \leq_{Q}\right) \subseteq P$ and $\operatorname{Bet}(Q)=\operatorname{Bet}(P)$. If $x<^{\prime} y$, then, we have $x<_{Q} y$ : to prove this, we observe that the definitions yield $x<y<z$ or $z<x<y$ or $x<z<y$ of some $z$. In the first case, $(x, y, z) \in B_{P}=B_{Q}$, hence $x<_{Q} y<_{Q} z$ because $Q \subseteq P$, and so $x<_{Q} y$. The proofs are similar for the two other cases. Hence $\widetilde{P} \subseteq Q$ and $\widetilde{P}$ is the unique minimal poset $Q$ such that $\operatorname{Bet}(Q)=\operatorname{Bet}(P)$ and $Q \subseteq P$.

An element that is not isolated in $P$ may be isolated in $\widetilde{P}$.

A poset $P$ is $B$-reconstructible (that is reconstructible from its betweenness relation) if $P$ and $P^{\text {rev }}$ are the only ones whose betweenness structure is $\operatorname{Bet}(P)$. A poset of cardinality 2 is not B-reconstructible. If $\widetilde{P} \subset P$, then $P$ is not reconstructible, because there are $x, y$ in $V$ such that $x<y, x \in \operatorname{Min}(P)$ and $y \in \operatorname{Max}(P)$ and $x<z<y$ for no $z$. Then $\operatorname{Bet}(\widetilde{P})=\operatorname{Bet}(P)=\operatorname{Bet}\left(P^{r e v}\right)$ but $\widetilde{P} \neq P^{r e v}$ and $\widetilde{P} \neq P$.

Two partial orders $\leq$ and $\leq^{\prime}$ defined on a set $V$ agree on $X \subseteq V$ if they induce on $X$ the same partial order.

Lemma 3.4. (1) Let $L=(V, \leq)$ be a linear order and $a<b$. Then $\leq$ is the unique order ${ }^{5}$ whose betweenness relation is $B_{L}$ and such that $a<b$.

(2) Let $P=(V, \leq)$ be a partial order. Let $C$ and $D$ be two maximal chains of cardinality at least 3 such that $C \cap D \neq \emptyset$. Let $Q=\left(V, \leq^{\prime}\right)$ be a partial order whose betweenness relation is $B_{P}$ and such that $\leq^{\prime}$ and $\leq$ agree on $C$. Then, $\leq$ and $\leq$ also agree on $D$.

Proof. (1) If $|V| \leq 2$, then $B_{L}$ is empty and the result holds. Otherwise, let $\leq^{\prime}$ be a partial order on $V$ whose betweenness relation $B^{\prime}$ is equal to $B_{L}$. The relation $B^{\prime}$ satisfies properties B1-B6, hence $\leq^{\prime}$ is a linear order. Proposition 5.3 of [5] proves that it is the same as $\leq$.

(2) Let $P, C, D$ and $Q$ be as in the statement. The order $\leq$ induces on $D$ a linear order because its betweenness relation equal to $B_{P}$ satisfies properties B1-B6 on $D$ and $|D| \geq 3$. If $|C \cap D| \geq 2$, the claim follows from (1) because we have $a<b$ and $a<^{\prime} b$ for some $a, b \in C \cap D$.

Otherwise, let $\{a\}=C \cap D$. By the maximality conditions on $C$ and $D$, we can have three cases.

(i) $a=\operatorname{Max}(C)=\operatorname{Max}(D)$,

(ii) $a=\operatorname{Min}(C)=\operatorname{Min}(D)$,

(iii) $a$ is extremal neither in $C$ nor in $D$.

\footnotetext{
${ }^{5}$ This order is $\mathrm{FO}$ definable in the structure $\left(V, B_{L}, a, b\right)$.
} 
In Case (i) we have $b \in C$ and $c \in D$ such that $b<a, b<^{\prime} a$ and $c<a$. We cannot have $a<^{\prime} c$ because we would have $b<^{\prime} a<^{\prime} c$, hence $(b, a, c) \in B_{Q}=B_{P}, b<a<c$, and $a \notin \operatorname{Max}(C)$. Hence, $c<^{\prime} a$ and (1) shows that $\leq$ and $\leq$ agree on $D$. Case (ii) is proved similarly.

For Case (iii), we have $b \in C$ and $c \in D$ such that $b<a, b<^{\prime} a$ and $a<c$. Hence, we have $(b, a, c) \in B_{P}=B_{Q}$ and so $b<^{\prime} a<^{\prime} c$ or $c<^{\prime} a<^{\prime} b$. The first holds since $b<^{\prime} a$, hence by $(1), \leq^{\prime}$ and $\leq$ agree on $D$.

Theorem 3.5. (1) Let $P=(V, \leq)$ and $Q=\left(V, \leq^{\prime}\right)$ be B-minimal and connected posets such that $B_{P}=B_{Q}$. Then $Q=P$ or $Q=P^{r e v}$.

(2) A poset of cardinality at least 3 is B-reconstructible if and only if:

(2.1) either it is connected and strongly B-minimal,

(2.2) or it has two connected components, one that is an isolated element, and the other that has no extremal elements.

Proof. (1) Let $P$ and $Q$ be as in the statement. All maximal chains have cardinality at least 3 . Let $C$ be a maximal chain in $P$. By Lemma 3.4(1), $\leq$ induces on $C$ the linear order $\leq$ or its reversal. We assume the first. Otherwise, we continue the proof with $P^{r e v}$ instead of $P$.

Consider a sequence $C_{1}, \ldots, C_{n}$ of maximal chains in $P$ such that $C=C_{1}$ and $C_{i} \cap C_{i+1} \neq \emptyset$ for each $i<n$. Then Lemma 3.4(2) shows that $\leq^{\prime}$ and $\leq$ agree on $C_{i}$ for each $i=2, \ldots, n$.

Let $a<b$. We will prove that $a<^{\prime} b$. The elements $a$ and $b$ belong to a maximal chain in $P$. Since $\operatorname{Comp}(P)$ is connected, there is a sequence $C_{1}, \ldots, C_{n}$ of maximal chains as above such that $C=C_{1}$ and $a, b \in C_{n}$. Then, the previous observation shows that $\leq^{\prime}$ and $\leq$ agree on $C_{n}$. Hence, $a<^{\prime} b$ and so $P=(V, \leq) \subseteq Q=\left(V, \leq^{\prime}\right)$. By exchanging $P$ and $Q$, we get $Q \subseteq P$, hence an equality.

(2) We first consider connected posets and prove (2.1). Let $P=(V, \leq)$ be strongly B-minimal and $Q=\left(V, \leq^{\prime}\right)$ have the same betweenness relation as $P$. As in the proof of (1), we can fix a maximal chain $C$ on which $\leq$ and $\leq^{\prime}$ agree. All maximal chains in $P$ have cardinality at least 3 as $P$ is B-minimal.

Claim 1: $\leq$ and $\leq^{\prime}$ agree on every maximal chain in $P$.

Proof. Let $D$ be a maximal chain in $P$. Since $\operatorname{Comp}(P)$ is connected, there is a sequence $C_{1}, C_{2}, \ldots, C_{n}$ of maximal chains in $P$ such that $C_{i} \cap C_{i+1} \neq \emptyset$ for each $i, C=C_{1}$ and $D=C_{n}$. By Lemma $7(2), \leq$ and $\leq \prime$ agree on $C_{1}, C_{2}, \ldots, C_{n}$, hence on $D$.

Claim 2: $\leq$ and $\leq^{\prime}$ agree on $V$.

Proof. If $a<b$, then $a$ and $b$ belong to a maximal chain in $P$, hence, by Claim 1 , we have $a<^{\prime} b$.

Conversely, let $a<^{\prime} b$. If $a$ and $b$ belong to a maximal chain in $P$, then $a<b$ or $b<a$. As $\leq$ and $\leq^{\prime}$ agree on every maximal chain in $P$, we have $a<b$.

Otherwise, assume that $a \notin \operatorname{Min}(P)$. Hence $c<a$ for some $c$, and $c$ and $a$ belong to a maximal chain in $P$. By Claim 1, we have $c<^{\prime} a$. Hence $c<^{\prime} a<^{\prime} b$, and $(c, a, b) \in B_{Q}=B_{P}$. Hence, $a$ and $b$ belong to a maximal chain in $P$, contradicting the initial assumption. Hence, we have $a \in \operatorname{Min}(P)$. If $b \in \operatorname{Max}(P)$, then, since $P$ is strongly B-minimal, we have $a<c<b$ for some $c$. Hence $a$ and $b$ belong to a maximal chain in $P$, contradicting the initial assumption. So, we have $b<c$ for some $c$, and then, $b$ and $c$ belong to a maximal chain in $P$ and $b<^{\prime} c$. Hence, $a<^{\prime} b<^{\prime} c,(a, b, c) \in B_{Q}=B_{P}$, and $a$ and $b$ belong to a maximal chain in $P$, contradicting the assumption. We have proved that $a<^{\prime} b$.

Claim 2 shows that $P=Q$.

For the converse, assume that $P=(V, \leq)$ is not strongly minimal. There are $a \in \operatorname{Min}(P), b \in \operatorname{Max}(P)$ without any $c$ such that $a<c<b$. We can add or remove from $\leq$ the pair $(a, b)$, without changing the betweenness relation, and we still have a poset. This shows that $P$ is not B-reconstructible.

To prove $(2.2)$, we assume that $P=(V, \leq)$ is not connected. It is not B-reconstructible in the following cases.

(i) It has a connected component $X$ that is not B-reconstructible. This means that there is a partial order $\leq^{\prime}$ on $X$ such that $B_{\left(X, \leq^{\prime}\right)}=B_{P} \cap X^{3}$ and $\leq^{\prime}$ does not agree on $X$ with $\leq$ or its reversal. If we replace in $P$ 
the order $\leq$ on $X$ by $\leq^{\prime}$, we obtain an order on $V$ whose betweenness relation is $B_{P}$ but that differs from $P$ and $P^{r e v}$.

(ii) Two connected components are isolated elements $a$ and $b$. Letting $a<b, b<a$ or $a$ and $b$ incomparable does not change $B_{P}$. Hence, at least 3 orders on $V$ have a betweenness relation equal to $B_{P}$.

(iii) Two connected components that are not isolated elements are B-reconstruc-tible. Then, at least 4 orders on $V$ have a betweenness relation equal to $B_{P}$. (Note that if a poset is not trivial, then it differs from its reversal).

It remains to consider the case where $P=(V, \leq)$ has two connected components, one that is an isolated element $a$ and the other one, $X$, that is B-reconstructible.

If $X$ has a maximal (resp. minimal) element $b$, then adding to $\leq$ the pair $(a, b)$ (resp. $(b, a)$ ) does not change the betweenness relation and we still have a poset. Hence, $P$ is not B-reconstructible.

If conversely, $P$ has no extremal element, adding $(a, b)$ or $(b, a)$ to $\leq$ does change the betweenness relation: if we add $(a, b)$, we have $b<c$ for some $c$, and $B_{P}$ becomes augmented with all triples $(a, b, d)$ for all $d>b$; the proof is similar if we add $(b, a)$. Hence, apart from reversing the order of $P$, any change to it will change the betweenness relation. Hence $P$ is B-reconstructible.

This completes the proof of $(2.2)$ and that of the theorem.

\subsection{A monadic second-order axiomatization of the class $\mathcal{B}_{P O}$}

We let $\mathcal{B}_{P O}$ be the class of betweenness structures $\operatorname{Bet}(P)$ for finite or countably infinite posets $P$.

Theorem 3.6. The class $\mathcal{B}_{P O}$ is $M S O$ axiomatizable among finite or countably infinite ternary structures. There is a pair of monadic second-order formulas that defines, for each $S \in \mathcal{B}_{P O}$, some partial order $P$ such that $S=\operatorname{Bet}(P)$.

Definition 3.7. Cut of a partial order.

A cut of a poset $P=(V, \leq)$ is a partition $(L, U)$ of $V$ such that:

(i) $L$ is downwards closed and $U$ is upwards closed,

(ii) Every maximal chain meets $L$ and $U$.

Note that $(U, L)$ is a cut of the reversal $(V, \geq)$ of $P$. These cuts are Dedekind cuts in linear orders.

Lemma 3.8. [9] : Every poset $P$ without isolated element has a cut.

Proof. We let $A$ be a maximal antichain of $P$. There exists one that is constructible from an enumeration ${ }^{6}$ $v_{1}, v_{2}, \ldots$ of $V$. We define

$$
U:=\{x \in V-\operatorname{Min}(P) \mid x \geq y \text { for some } y \in A\} \text { and } L:=V-U .
$$

We prove that $(L, U)$ is a cut. From the definition, $U$ is upwards closed, and so, $L$ is downwards closed. Let $C$ be a maximal chain: it contains a unique element $a \in A$. If $a=\operatorname{Min}(C)$, then $a \in \operatorname{Min}(P) \subseteq L$. As $a$ is not isolated in $P, a \neq \operatorname{Max}(C)$, hence, we have $a<x$ for some $x \in C$, and so $x \in U$. Otherwise, we have $y<a$ for some $y \in C$, hence $a \notin \operatorname{Min}(P)$ and so $a \in U$ and $y \notin U$. Hence, $(L, U)$ is a cut.

Proof of Theorem 3.6. It follows from Proposition 6 that $\mathcal{B}_{P O}$ is the class of structures $\operatorname{Bet}(P)$ for B-minimal posets $P$.

First part: We first characterize the structures $\operatorname{Bet}(P)$ for B-minimal posets $P$ without isolated elements.

Let $P=(V, \leq)$ be such a poset and $(L, U)$ be a cut of it. We claim that $\leq$ can be defined from $L, U$ and the betweenness relation $B_{P}$ by $\mathrm{FO}$ formulas ${ }^{7}$.

\footnotetext{
${ }^{6}$ We consider finite or countably infinite sets that are effectively given, see [8], hence, that have some explicit or implicit enumeration. The Choice Axiom can also be used to assert the existence of an enumeration.

${ }^{7}$ We allow free set variables, here $L$, in FO formulas. We make explicit the dependence on the relation $B$.
} 
Claim 1: For $x, y \in V$, we have $x<y$ if and only if one of following conditions holds, for $B:=B_{P}$ :

(i) $x \in L, y \in U$, and $B(x, y, z) \vee B(x, z, y) \vee B(z, x, y)$ holds for some $z$,

(ii) $x, y \in L$ and $B(x, y, w)$ holds for some $w \in U$,

(iii) $x, y \in U$ and $B(w, x, y)$ holds for some $w \in L$.

Proof. Let $x, y$ be such that $x<y$. As $P$ is B-minimal, $x$ and $y$ belong to a maximal chain $C$ of cardinality at least 3. This chain contains some $z$ such that $x<y<z, x<z<y$ or $z<x<y$ and meets $L$ and $U$.

If $x \in L$ and $y \in U$, then each of these three cases can hold and yields respectively $B(x, y, z), B(x, z, y)$ or $B(z, x, y)$. If $x, y \in L$, then, since $C$ meets $U$, we have some $w \in U$ such that $x<y<w$ and $B(x, y, w)$ holds. If $x, y \in U$ then, since $C$ meets $L$, we have some $w \in L$ such that $w<x<y$ and $B(w, x, y)$ holds. Hence, we have one of the exclusive cases (i), (ii) or (iii).

Let $\varphi(B, L, x, y)$ be the FO formula expressing the disjunction of Conditions (i), (ii) and (iii) in a ternary structure $S=(V, B)$, where $L \subseteq V$, and $U$ is defined as $V-L$. (If B1 holds, these conditions imply $x \neq y$ ). Then, there exists an FO formula $\psi(B, L)$ expressing the following:

(a) $B$ satisfies properties B1, B2 and B3, and every element of $V$ belongs to some triple in $B$.

(b) $L$ and its complement $U:=V-L$ are not empty,

(c) the binary relation $\{(x, y) \in V \times V \mid S \models \varphi(B, L, x, y)\}$ is a strict partial order $<$ that is B-minimal ${ }^{8}$,

(d) $B$ is the betweenness relation of $<$.

Finally, we let $\theta(B)$ be the MSO sentence $\exists L \cdot \psi(B, L)$.

Claim 2: For a ternary structure $S=(V, B)$, we have $S \models \theta(B)$ if and only if $S=\operatorname{Bet}(P)$ for a B-minimal poset $P=(V, \leq)$ and $S$ has no isolated elements.

Proof. "If" Let $P=(V, \leq)$ be B-minimal, $B=B_{P}$ be its betweenness relation, and assume that $\operatorname{Bet}(P)$ has no isolated elements. The poset $P$ has none either and has a cut $(L, U)$. Properties (a) and (b) hold by the definitions. By Claim 1, $\varphi(B, L, x, y)$ defines the strict partial order < and so, (c) and (d) hold. So we have $S \models \theta(B)$.

Conversely, assume that $B$ and $L$ satisfy $\psi(B, L)$. Properties (c) and (d) hold hence $B=B_{P}$ for the strict and B-minimal partial order $P$ defined by $\varphi(B, L, x, y)$. By Property (a), $S$ has no isolated elements.

To define a B-minimal partial order $P$ such that $\operatorname{Bet}(P)=S$ where $S$ satisfies $\theta(B)$, we use the following MSO formulas:

$\psi(B, L)$ intended to select in $S=(V, B)$ an appropriate set $L$.

$\varphi(B, L, x, y)$ that defines the partial order in terms of $L$ assumed to satisfy $\psi(B, L)$.

If $P$ is a partial order such that $\operatorname{Bet}(P)$ has no isolated elements, then, $\operatorname{Bet}(P) \models \theta(B)$ because, by Proposition 3.3, $\operatorname{Bet}(P)=\operatorname{Bet}(\widetilde{P})$ where $\widetilde{P}$ is B-minimal. The formula $\varphi(B, L, x, y)$ defines, in the structure $\operatorname{Bet}(P)$, partial orders $Q$ such that $\operatorname{Bet}(Q)=\operatorname{Bet}(P)$ (but $Q$ may differ from $P$ ). Note that $P$ need not be connected.

Second part: Let be given a structure $S=(V, B)$. It is the union of its connected components $S[X]$ where the sets $X$ are the vertex sets of the connected components of $G f(S)$. There is nothing to verify for the components which are isolated elements. The others can identified by an MSO formula $\gamma(X), c f$. [8]. Then, $S \in \mathcal{B}_{P O}$ if and only if each of these components satisfies $\theta(B)$. For this purpose, we translate $\theta(B)$ into a formula $\theta^{\prime}(B, X)$ such that, for every subset $X$ of $V$,

$$
S \models \theta^{\prime}(B, X) \text { if and only if } S[X] \models \theta\left(B \cap X^{3}\right) .
$$

This is a classical construction called relativization of quantifications to a set $X$, see e.g. [8]. Hence, a structure $S$ belongs to $\mathcal{B}_{P O}$ if and only if:

$$
S \models \forall X .\left(\gamma(X) \Longrightarrow \theta^{\prime}(B, X)\right) .
$$

\footnotetext{
${ }^{8}$ We mean that the associated partial order $\leq$ is B-minimal.
} 
Similarily, $\psi(B, L)$ can be transformed into $\psi^{\prime}$ such that, if $X, L \subseteq V$, then:

$$
S \models \psi^{\prime}(B, L \cap X, X) \text { if and only if } S[X] \models \psi\left(B \cap X^{3}, L \cap X\right) .
$$

It follows that, from a set $L \subseteq V$ such that:

$$
S \models \forall X .\left(\gamma(X) \Longrightarrow \psi^{\prime}(B, L \cap X, X)\right),
$$

one can define partial orders for the components $S[X]$.

We transform $\varphi(B, L, x, y)$ into $\varphi^{\prime}(B, L, X, x, y)$ that defines a partial order on $X$, by using $L \cap X$. Then, from $L$ as above, one obtains a strict partial order on $V$ defined by:

$$
x<y: \Longleftrightarrow S \models \exists X .\left(x, y \in X \wedge \gamma(X) \wedge \varphi^{\prime}(B, L, X, x, y)\right)
$$

whose betweenness relation is $B$. This completes the proof of the theorem.

Remark 3.9. A B-minimal poset $P=(V, \leq)$ has several cuts $(L, U)$. However, from the structure $\operatorname{Bet}(P)$, they yield only two orders whose betweenness relation is $\operatorname{Bet}(P)$, provided $P$ is connected. If $G f(\operatorname{Bet}(P))$ has $n$ connected components that are not singleton, the formulas $\psi^{\prime}$ and $\varphi^{\prime}$ define $2^{n}$ partial orders whose betweenness structure is $\operatorname{Bet}(P)$.

Remark 3.10. A partial order is reconstructible, u.t.r., from its comparability graph if and only if this graph is prime. This notion is relative to the theory of modular decomposition. Furthermore, there is an MSO formula that defines the two transitive orientations of a prime comparability graph $G$, equivalently, the two partial orders $P$ such that $G=\operatorname{Comp}(P)$. More generally, the class of comparability graphs is MSO definable, and primality is MSO definable. These results are proved in Section 5 of [3]. They concern finite and countably infinite partial orders.

\section{Finite PARTial ORDERS}

We give an algorithm that decides in polynomial time whether a finite ternary structure $S=(V, B)$ is $\operatorname{Bet}(P)$ for some poset $P$, and produces one if possible.

Lemma 4.1. Let $P=(V, \leq)$ is a finite and B-minimal poset. For $x, y \in V$, we have $x<y$ if and only if:

either $B_{P}(x, y, z)$ holds for some $z \in \operatorname{Max}(P)$, or $y \in \operatorname{Max}(P)$ and $B_{P}(w, x, y) \vee B_{P}(x, w, y)$ holds for some $w \in V$.

Proof. The "if" direction is clear, since $\operatorname{Max}(P)$ is not empty.

For the converse, assume that $x<y$. The elements $x$ and $y$ belong to a chain of cardinality at least 3 with maximal element $z \in \operatorname{Max}(P)$. If $y \neq z$ we have $B_{P}(x, y, z)$, otherwise $B_{P}(w, x, y) \vee B_{P}(x, w, y)$ for some $w \in V$. (The term $B_{P}(x, w, y)$ is for the case where $x$ is minimal).

We recall that in a ternary structure $S=(V, B)$ that satisfies Properties B1, B2 and B3, an element $x \in V$ is extremal if $B(y, x, z)$ does not hold for any $y, z$. We let $\operatorname{EXT}(S)$ be the graph whose vertex set is the set of extremal elements denoted by $\operatorname{Ext}(S)$ and $u-v$ is an edge if and only if $B(u, w, v)$ holds for some $w$ (necessarly not in $\operatorname{Ext}(S))$. It follows that $\operatorname{EXT}(S)$ is a subgraph of $G f(S)$ and of $\operatorname{Comp}(P)$ if $S=\operatorname{Bet}(P)$.

Lemma 4.2. Let $P=(V, \leq)$ be a finite and B-minimal partial order without isolated elements. The graph $\operatorname{EXT}(\operatorname{Bet}(P))$ is bipartite with bipartition $(\operatorname{Max}(P), \operatorname{Min}(P))$. It is connected if $P$ is.

Proof. Each element of $\operatorname{Max}(P) \cup \operatorname{Min}(P)$ is extremal in $\operatorname{Bet}(P)$. If $w \notin \operatorname{Max}(P) \cup \operatorname{Min}(P)$ then $u<w<v$ for some $u, v$ and so $B_{P}(u, w, v)$ holds and $w$ is not extremal in $\operatorname{Bet}(P)$.

The set $\operatorname{Max}(P) \cap \operatorname{Min}(P)$ is empty. If $u-v$ is an edge of $\operatorname{EXT}(\operatorname{Bet}(P))$, then $B_{P}(u, w, v)$ holds, hence $u<w<v$ or $v<w<u$ and $u$ and $v$ cannot be both in $\operatorname{Max}(P)$ or in $\operatorname{Min}(P)$. Hence, $\operatorname{EXT}(\operatorname{Bet}(P))$ is bipartite with bipartition $(\operatorname{Max}(P), \operatorname{Min}(P))$. 
Assume now that $P$ is connected. Let $x, y \in \operatorname{Ext}(P)$ and $x-z_{1}-\ldots-z_{n}-y$ be a shortest path in $\operatorname{Comp}(P)$. We claim that there exists also one $x-u_{1}-\ldots-u_{n}-y$ such that $u_{1}, \ldots, u_{n}$ are extremal. Without loss of generality, we assume that $x \in \operatorname{Min}(P)$. If $z_{1}<z_{2}$, then $x-z_{2}$ is an edge in $\operatorname{Comp}(P)$ and the considered path is not shortest between $x$ and $y$. Hence, $z_{1}>z_{2}$. Let $u_{1} \in \operatorname{Max}(P)$ such that $z_{1} \leq u_{1}$. Hence $u_{1}>z_{2}$ and we have a path $x-u_{1}-z_{2}-\ldots-y$ in $\operatorname{Comp}(P)$. Similarly, we cannot have $z_{3}<z_{2}$. We let $u_{2} \in \operatorname{Min}(P)$ be such that $u_{2}<z_{2}$. We have a path $x-u_{1}-u_{2}-z_{3} \ldots-y$ in $\operatorname{Comp}(P)$. By continuing in this way, we obtain a path $x-u_{1}-\ldots-u_{n}-y$ such that $u_{1}, \ldots, u_{n}$ are extremal. Hence $\operatorname{EXT}(\operatorname{Bet}(P))$ is connected. Note that this path we have an alternation of minimal and maximal elements.

This proof shows in particular that the odd $B$-cycles are not $B e t(P)$ for any partial order $P$, because their extremal elements are $a_{1}, \ldots, a_{n}$ (cf. Def. 2.1(c)) forming an odd cycle and $\operatorname{EXT}(\operatorname{Bet}(P))$ would not be bipartite.

Theorem 4.3. There exists a polynomial time algorithm that decides whether a finite ternary structure $S=$ $(V, B)$ is the betweenness structure of a partial order $P$, and produces one if possible.

Proof. Let be given a finite ternary structure $S=(V, B), n:=|V|$ and $m:=|B|=O\left(n^{3}\right)$.

Step 1 : In order to eliminate trivial cases, the algorithm first checks Properties B1,B2 and B3, and if they hold, it constructs the graph $G f(S)$ and determines its connected components. This step takes time $O(n+m)$.

Each connected component is then considered and still denoted by $S$.

Step 2 : The algorithm constructs the graph $\operatorname{EXT}(S)$, checks if it is bipartite and if it is, it determines its bipartition $\left(V_{1}, V_{2}\right)$ that is unique because $\operatorname{EXT}(S)$ is connected. If $\operatorname{EXT}(S)$ is not bipartite, the algorithm stops and returns a negative answer. This step takes time $O(m)$.

Step 3 : By taking $V_{1}$ as intended set of maximal elements, the algorithm defines a binary relation $<$ as follows:

$x<y$ if and only if $B(x, y, z)$ holds for some $z \in V_{1}$,

or $y \in V_{1}$ and $B(w, x, y) \vee B(x, w, y)$ holds for some $w \in V-V_{1}$.

This step take time $O(m)$. If $S=\operatorname{Bet}(P)$ for some poset $P$, then, by Lemma $4.1,<$ is a B-minimal strict partial order such that $\operatorname{Bet}(Q)=S$, where $Q:=(V, \leq)$

Step 4 : The algorithm verifies that $B=\operatorname{Bet}(Q)$. This step takes time $O\left(n^{3}\right)$. If $B \neq \operatorname{Bet}(Q)$, it can report a failure.

This algorithm can perhaps be improved by means of clever data-structures.

\section{No Finite FirST-ORDER AXIOMATIZATION OF $\mathcal{B}_{P O}$}

Proposition 5.1. The class $\mathcal{B}_{P O}$ is not $F O$ axiomatizable, i.e., is not the class of finite or countably infinite models of a single first-order sentence.

We recall that it is by the conjunction of an infinite set of universal first-order sentences [10].

Proof. The proof is based on a reduction to a first-order definability result for languages.

A word $w$ of length $p>0$ over the alphabet $\{a, b\}$ can be represented by the relational structure $S(w):=$ $([p], \leq, A)$ whose domain $[p]:=\{1,2, \ldots, p\}$ is the set of positions of letters, $\leq$ is the standard order and $A \subseteq[p]$ is the set of positions of letter $a$. Hence $B:=[p]-A$ is the set of positions of $b$. We let $\operatorname{suc}(x, y)$ express that $y$ is the position following $x$; this relation is FO definable from $\leq$.

The language $K:=\left\{(a b)^{m} \mid m \geq 2\right\}$ is FO definable, which means that there exists an FO sentence $\kappa$ such that, for every nonempty word $w$ over $\{a, b\}$, we have $w \in K$ if and only if $S(w) \models \kappa$.

We define an FO formula $\beta(x, y, z)$ relative to $S(w)$ that expresses the following property of a triple $(x, y, z)$ of positions of a word $w$ in $K$ :

$x \in A \wedge \operatorname{suc}(x, y) \wedge \operatorname{suc}(y, z)$ or

$z \in A \wedge \operatorname{suc}(z, y) \wedge \operatorname{suc}(y, x)$ or

$x \in A \wedge \operatorname{suc}(z, y), x$ is the first position of $w$ and $y$ is the last one or 
$z \in A \wedge \operatorname{suc}(x, y), z$ is the first position of $w$ and $y$ is the last one.

These triples form a set $B_{w} \subseteq[2 m]^{3}$ (that satisfies Properties B1,B2 and B3). The structure $T(w):=$ $\left([2 m], B_{w}\right)$ is a $B$-cycle as in Definition 2.1(c). Its vertices $a_{1}, \ldots, a_{m}$ are the odd positions, those of letter $a$, its vertices $b_{1}, \ldots, b_{m}$ are the even positions, those of letter $b$. It follows from Definition 2.1(c) and Lemma 4.2 that $T(w) \in \mathcal{B}_{P O}$ if and only if $m$ is even.

Assume now that $\mathcal{B}_{P O}$ is axiomatized by a first-order sentence $\theta$, possibly not universal. This sentence can be translated into an FO sentence $\theta^{\prime}$ such that, for every word $w$ over $\{a, b\}$, we have:

$S(w) \models \kappa \wedge \theta^{\prime}$

if and only if $w \in K$ and $T(w) \models \theta$

if and only if $w \in K$ and $T(w) \in \mathcal{B}_{P O}$

if and only if $w=(a b)^{2 n}$ for some $n$.

The language $L:=\left\{(a b)^{2 n} \mid n \geq 1\right\}$ would be first-order definable, which is not the case by a classical result due to McNaughton, Papert and Schützenberger, see [11].

We get a contradiction hence, the class $\mathcal{B}_{P O}$ is not axiomatizable by a single first-order sentence.

Acknowledgements. I thank Maurice Pouzet for his comments from which I obtained the easy proof of Proposition 5.1.

\section{REFERENCES}

[1] M. Changat, P. Narasimha-Shenoi and G. Seethakuttyamma, Betweenness in graphs: A short survey on shortest and induced path betweenness. AKCE Int. J. Graphs Combinat. 16 (2019) 96-109.

[2] V. Chvatal, Antimatroids, betweenness, convexity, in Research Trends in Combinatorial Optimization, Spriner (2008) 57-64.

[3] B. Courcelle, The monadic second-order logic of graphs XV: On a conjecture by D. Seese. J. Appl. Log. 4 (2006) $79-114$.

[4] B. Courcelle, Several notions of rank-width for countable graphs, J. Combin. Theory B 123 (2017) 186-214.

[5] B. Courcelle, Algebraic and logical descriptions of generalized trees, Logical Methods in Computer Science 13 (2017) 7.

[6] B. Courcelle, Axiomatization of betweenness in order-theoretic trees, February (2019). https://hal.archives-ouvertes.fr/ hal-02205829, to appear in Logical Methods in Computer Science (2020).

[7] B. Courcelle, Betweenness in order-theoretic trees, in Fields of Logic and Computation III, Lecture Notes in Computer Science 12180 (2020) 79-94.

[8] B. Courcelle and J. Engelfriet, Graph structure and monadic second-order logic, a language theoretic approach. Cambridge University Press (2012).

[9] D. Duffus, V. Rödl, N. Sauer and R. Woodrow, Coloring ordered sets to avoid monochromatic maximal chains. Canad. J. Math. 44 (1991) 91-103.

[10] J. Lihova, Strict-order betweenness. Acta Univ. M. Belii Ser. Math. 8 (2000) 27-33.

[11] W. Thomas. Classifying regular events in symbolic logic. J. Comput. Syst. Sci. 25 (1982) 360-376 